\title{
Improvement of tribotechnological properties of oil-based cutting fluids by adding dry lubricants
}

\author{
Dilyaver Abdulgazis ${ }^{1}$, Ervin Umerov $^{1, *}$ and Umer Abdulgazis ${ }^{1}$ \\ ${ }^{1}$ Crimean Engineering and Pedagogical University, 295015, Lane training, 8, Simferopol, Republic of Crimea, Russian Federation
}

\begin{abstract}
Having taken an oil component of cutting fluids used when machining difficult-to-machine materials, authors have searched for an additive which would have improved both tribotechnological properties and the cooling effect. Tribotechnological characteristics of widely used friction modifiers, such as graphite, molybdenum disulfide and serpentine, are analyzed. It has been show that the use of montmorillonite, a mineral that is widespread in nature, as an additive improves tribotechnological characteristics and scuffing load capacity of oil-based cutting fluids in general.
\end{abstract}

\section{Problem statement}

Basic properties required from oil-based cutting fluids are the improvement of hardness of a cutting instrument as well as the conditions of a working surface. Thanks to this, oilbased cutting fluids more or less determine costeffectiveness and reliability of metal processing equipment. Oftentimes it is difficult to discriminate between the most important effects of oil-based cutting fluids. Edge-cutting machining of difficult-to-machine materials poses more significant requirements to both cooling and lubricating effect of oil-based cutting fluids [1].

At the same time, the technological tools currently used exhibit either the former or the latter effect, which prompts specialists in the field to search for an additive agent, which would have had the ability to improve tribotechnological properties and the cooling effect, taking oil-based cutting fluids as a basic material used during machining of difficult-to-machine materials. Therefore, an additive agent to an oil-based cutting fluid should exhibit endothermic and tribotechnological properties

\section{Literature review}

This double function necessary from an additive agent requires doing the search among non-traditional materials.

A promising trend in lubricant research has been the search for various kinds of fillers and additives, primarily solid and naturally occurring, which would have improved tribotechnological properties of liquid and grease lubricants. We believe that the search of endothermic properties is of equal importance.

Of all known endothermic processes which may be used to improve the cooling ability of oil-based cutting fluids, endothermic effect of crystalline hydrates was considered to be the most viable [2-5]. According to our analysis, addition of crystalline hydrates to oil-based cutting fluids does not improve their tribotechnological properties.

Since additives to oil lubricants, including engine oil, may have the same function that the additives to oilbased cutting fluids have, we will consider the improvements in this area as well. For instance, modern engine oils use additives which decrease friction coefficient; among those additives, graphite-based and molybdenum greases are the most popular ones.

Unique tribotechnological properties of graphite and molybdenum disulfide are attributed to a flat hexagonal structure that their crystal lattices possess. The distance between the closest atoms of graphite is $1.42 \AA$, while the distance between the closest planes is $3.4 \AA$ [6]. Therefore, every crystal plane may be looked at as an isolated system of atoms. Atomic planes of graphite crystals are located parallel relative to one another.

Each of these planes consists of carbon atoms bonded covalently to each other to form regular hexagons. Adjacent layers are held together by weak van der Waals' forces, which are three to four times weaker than the covalent forces. This allows the layers to slide on each other [6].

Shear strength of graphite along crystal planes is incommensurably less than that of the graphite when covalent hexagonal bonds are broken. Additional lubricating effect of graphite is based on the fact that by sorbing between its crystal planes at normal temperature, water molecules relax interplanar bonds. Consequently, when the temperature is above $100^{\circ} \mathrm{C}$ thanks to evaporation effect, graphite greases exhibit reduced lubricating properties. In the absence of water, friction coefficient of surfaces divided by graphite layer may be as large as 0.3 , while in the presence of sorbate water [7] it is approximately 0.05 . This is the reason why graphite greases are used sparingly.

* Corresponding author: ervin777@yandex.ru 
Boron nitride may form the same structure where atoms of boron and nitrogen alternate [6].

Molybdenum disulfide $\left(\mathrm{MoS}_{2}\right)$, which possesses similar hexagonal crystal lattice, is characterized by stable chemical bonds between molybdenum atoms, while sulfur atoms have stable bonds with molybdenum atoms form the structure which connects adjacent layers of molybdenum atoms to each other via weak van der Waals forces. Thus, relatively small shear strength is formed at the border between layers of sulfur atoms. Presence of water in this case exerts little influence, therefore, $\mathrm{MoS}_{2}$ may be used at relatively high temperatures (up to $1000^{\circ} \mathrm{C}$ ). However, friction between its crystal lattices takes place in the absence of water.

Our assessment has also showed that, during thermal degradation in the cutting zone, molybdenum disulfide is capable of producing sulfur which may interact with cutting tool's material and consequently decrease its wear resistance and deteriorate the quality of mechanical and chemical structure of a working surface.

In this respect, metal carbides (Mo, V, W) may act more positively. At high temperature and pressure values in the cutting zone, metal carbides may penetrate into the lattices on the surfaces of a cutting tool and a workpiece, which in fact may improve strength of these lattices. Furthermore, it may improve wear resistance of a cutting tool and perform nanopatterning of workpiece's surface using alloying components.

Production of powdered molybdenum disulfide and stabilization of its ratio in oil lubricants is a timeconsuming and complex process which is possible only at large production plants that have highly trained personnel and high-precision equipment at their disposal [7].

Another trend in the area of addition agents is [8] the application of natural fine-grain serpentine which may help to increase wear resistance of workpiece parts and decrease friction coefficient. Serpentines possess layered structure, are not soluble in oil products and do not react with them; plus, they are environmentally harmless. Paper [8] also underlines that natural serpentine contains a wide range of impurities, and in order for it to be used as an additive agent, it has to undergo enrichment (grinding, separation etc.), which drastically increases its production costs. Therefore, authors of [8] suggest replacing natural serpentine with the artificial one obtained using sol-gel method.

Action mechanism of serpentine as a friction modifier may be considered similar to that of the powdered molybdenum disulfide.

Considering the aforementioned limitations of dry lubricants like graphite, molybdenum disulfide and its alternatives, authors have strived to find nanomaterials with improved tribotechnological and (possibly) endothermic properties that may be used as an additive agent.

\section{Overview}

Bentonites, plate-shaped nanoclay minerals, have favorable physical and chemical properties. Among other well-known benefits that dry lubricants like molybdenum disulfide possess, it is also possible to provide fluid lubrication between layers of minerals via their hydro-wedging, which allows for improvement of tribotechnological properties.

The aforementioned has prompted researchers to conduct studies regarding viability of use of nanoclay mineral additives in oil-based cutting fluids, with the purpose of increasing hardness of a cutting tool and the quality of machining of difficult-to-machine materials. Application of nanoclay mineral additives instead of well-known friction modifiers (graphite, molybdenum disulfide, serpentine) helps to eliminate a range of problems:

1. Nanoclay minerals are widespread in nature; they may be found in Crimea, which gives them a huge advantage in the form of lower cost of production. They are obtained using the open-pit method and do not require highly qualified personnel and precision equipment.

2. Despite some similarities between the crystal lattices of molybdenum disulfide and bentonites, shearing of layers of the former happens in the absence of water, while in the case of nanoclay mineral additives it happens in the presence of hydrowedging, that is, in the presence of liquid friction between layers of the mineral which will eventually influence the tribotechnological and endothermic properties.

3. Necessity to use dispersants and stabilizing agents when adding molybdenum disulfide to engine oils thanks to their immeasurably large service life before replacement is eliminated if nanoclay mineral additives are added to oil-based cutting fluids.

One of the main components of bentonites is montmorillonite, which is a nanodisperse plate-shaped aluminosilicate consisting of blocks of nanominerals capable of swelling when hydrated. It creates hydrowedging areas between the layers. This phenomenon is what distinguishes tribotechnological properties of montmorillonite from those of the solid lubricants described above (Fig. 1).

Packages are the least structural component of bentonites in general and of montmorillonite in particular. The thickness of packages is $1.0-10 \mathrm{~nm}$ while linear dimensions are not more than $50-150 \mathrm{~nm}$. The distance between packages at swelling (hydration) can increase up to $2.14 \mathrm{~nm}[4,5]$. 


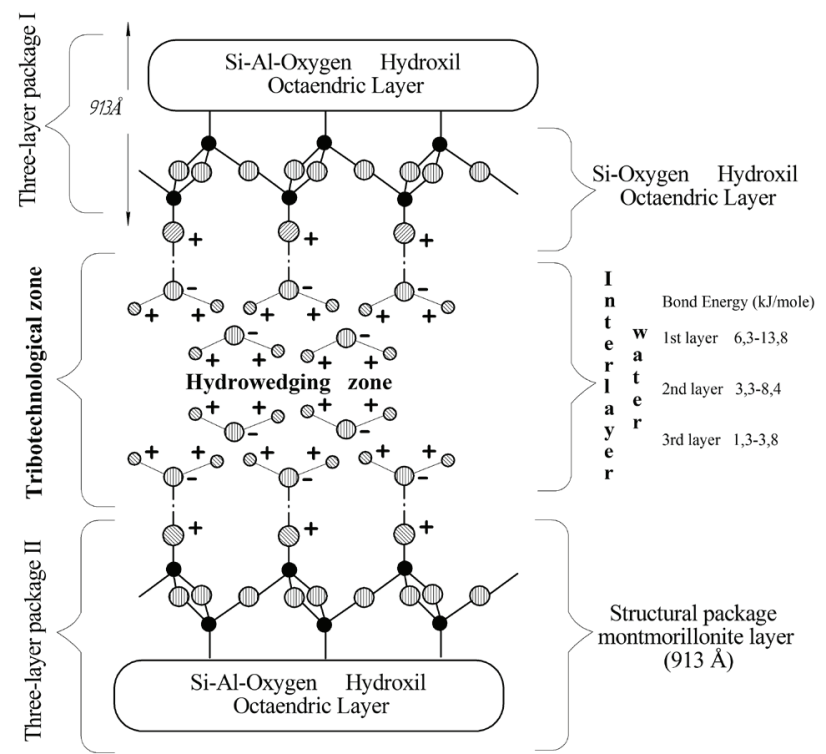

Fig. 1. The structural flow-chart of physical and chemical interaction of montmorillonite with water:

$$
\begin{gathered}
-\mathrm{OH} \text { hydroxyl; } \ominus-\mathrm{Al} \text { aluminum; }- \text { Si silicon; } \\
\mathbb{( D} \text { - O oxygen; } \mathbb{Q} \text { - H hydrogen }
\end{gathered}
$$

It shall be underlined that nanostructures have special properties intrinsic of nanolayers, nanocrystalls and nanoparticles connected to a so-called dimensional effect only. The property of the nanoclay mineral addition (NCMA) that is the most important to achieve the objective is the capacity of packages hydrowedging with superficially occluded water at hydration. As a result, it is possible to provide interpackage sliding due to occurring hydrolubrication thus improving tribotechnological properties of oil CLS in general (Figure 2).

It should be mentioned that recently modern motor oils have included additions modifying friction allowing for the decrease of the frictional factor between the rubbing engine parts. The most wide-known additions modifying friction include graphite and molybdenum disulphide. These unique properties are explained by the structure of their crystalline lattices. Binding between their layers is relatively weak similar to those of graphite and molybdenum disulphide and therefore it allows for the possible shifting at low voltage which determines decrease of the friction factor [6].

The use of NCMA for oil CLs eliminates a range of problems inherent in the use of molybdenum disulphide as an addition to motor oils:

1. Shift of molybdenum disulphide layers can be "dry" in NCMA; in case it is properly prepared in advance (hydrogenation), this shift can be accompanied by hydrolubrication, i.e. under fluid friction, which finally influences tribotechnical properties.

2. Nanoclay minerals are rather wide-spread natural mineral deposits. To prepare them for the procedure, one doesn't need to involve high-qualified staff and highprecision equipment.

3. There is also no need in dispersants and dispersion stabilizers which are required for molybdenum disulphide addition to motor oils. a)

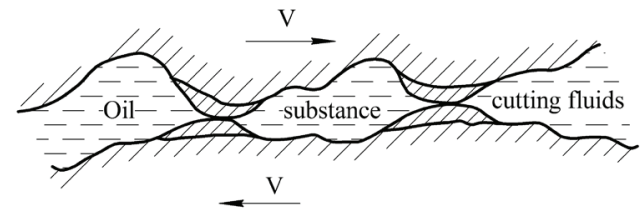

b)

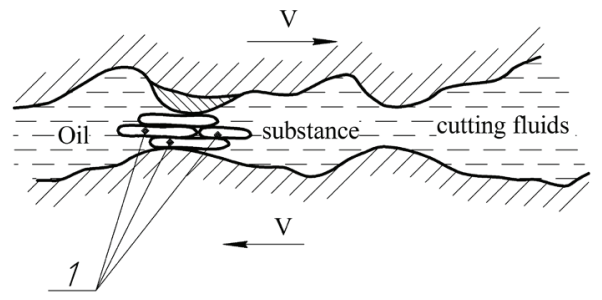

c)

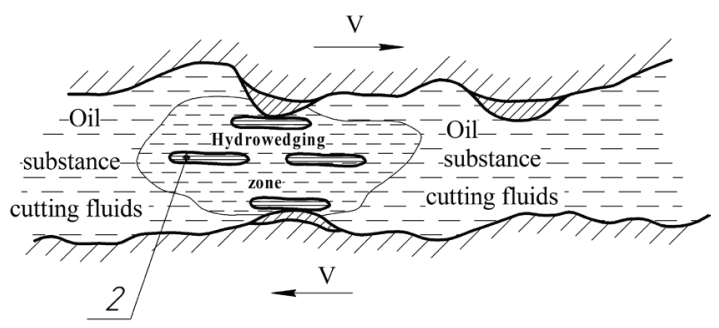

Fig. 2. Examples of implementation of junction and nanojunction coupling. Change of contact geometry: $a$ - of junction couplings (in the presence of dry junction);

$b-$ in the presence of molybdenum disulfide as an additive to oil lubricants;

$c-$ of nanojunction couplings, in the presence of montmorillonite as an additive to oil lubricants.

1 - crystal planes of either graphite or molybdenum disulfide;

2 - crystal blocks of montmorillonite;

EIJ - area of high local deformation;

$\mathrm{V}-$ linear speed.

Thermal action on the preliminarily wedged with superficially occluded water packages of nanominerals added to oil CLs in the cutting area leads to intense water evaporation providing extra endothermic reaction effect. Water vapours remain inside the system; when the temperature decreases, they return to its structure, and this is one of the properties characteristic of nanoclay minerals. As oil cannot interact with water, when the temperature lowers and the CL goes out from the cutting area, vapour is condensed and returns to the mineral structure recovering its cooling capacity.

The described process works similar to hydrationdehydration of crystalline hydrates described in the work [2]. Crystalline hydrates contain water in the defects of a crystalline lattice, and separating the first molecule requires bigger energy comparing to NCMA. Nanoclay minerals demonstrate the dehydration effect in a far more active way. The thermodynamic analysis [7] provides evidence that the endothermic effect of montmorillonite generally occurs in the temperature range of $50-200{ }^{\circ} \mathrm{C}$ with a maximum of $99{ }^{\circ} \mathrm{C}$.

At heating of the adsorptive water layer of a nanoclay mineral particle up to the temperature $80-100{ }^{\circ} \mathrm{C}$ the weakest molecular binds are broken up first which triggers the first hydration step. Further, as the temperature increases up to $100-200{ }^{\circ} \mathrm{C}$, medium strength molecular bind break up at the second turn, and 
when the temperature is more than $200{ }^{\circ} \mathrm{C}$, the stronger molecular binds break up next, etc., thus creating a stepwise dehydration endothermic process.

Therefore, NCMA can be used as CLs endothermic component similar to crystalline hydrates. But as opposed to the latter, NCMA is more thermally and chemically stable which excludes a possibility of its adhering and burning on the surfaces of the cutting tool and machine at high temperatures.

The fact that NCMA can show an endothermic effect similar to crystalline hydrates is confirmed by bentonite thermogram given in the work [8]. It confirms that at $100-150{ }^{\circ} \mathrm{C}$ the first endothermic effect step takes place - i.e., elimination of adsorbing and interpackage water. The closing step takes place at the temperature of 500$650{ }^{\circ} \mathrm{C}$ with elimination of a hydroxyl group.

If necessary, temperature discontinuity between the first and closing steps of the endothermic reaction can be filled with similar effects provided by other mineral or crystalline hydrates.

The comparison of the temperature range of NCMA dehydration on the basis of montmorillonite with a working temperature of CLs in the cutting area that was measured experimentally $[9,10]$ by means of cutter washing at various cutting modes with the flow of 0.15 $1 /$ min., shows that they are rather comparable and are equal to $76 \ldots 221^{\circ} \mathrm{C}$. According to [11], CLs temperature, for example, at steel workpiece drilling with a drill of 50 size out of a rapid steel R6M3 can reach $270{ }^{\circ} \mathrm{C}$.

Let us consider the opportunity of re-use or multiple use of oil CLs with NCMA. In this case a decisive meaning is attributed to the capacity of the considered addition of water re-absorption after the previous heating up to CLs working temperatures in the cutting area.

Basing upon the work [8], one may state that the previous heating mode can significantly change the following water absorption properties of NCMA. To confirm the above said, let us give a brief method and some results of the conducted experiment. Adding water to a NCMA sub-sample, we made it a paste-like, and after that the sub-sample was gradually dried at 200, 300, 400, 500, 600, 800 and $900^{\circ} \mathrm{C}$. After each drying stage and 10-minute temperature stabilization the sub-sample was re-hydrated.

Experiments demonstrated that NCMA heating up to the temperature $200{ }^{\circ} \mathrm{C}$ allows it to preserve its rehydration properties by $61 \%$. The most significant deterioration of NCMA re-hydration capacity is registered after its heating up to $500{ }^{\circ} \mathrm{C}$. At the same time, NCMA re-hydrates, i.e. preserves the necessary properties. only by $37 \%$. Such temperature threshold is rather satisfactory in terms of NCMA use in CLs as red hardness of rapid steel is $500^{\circ} \mathrm{C}$. At further heating up to 800-900 ${ }^{\circ} \mathrm{C}$, NCMA virtually loses its re-hydration capacity, and thus, it becomes unsuitable for re-use as an addition to CLs.

Summing up the aforementioned, one can assume that NCMA overheating deteriorates its properties. The temperature limit can be considered $500{ }^{\circ} \mathrm{C}$ while admissible thermal optimum for endothermic effect of NCMA in CLs is $200{ }^{\circ} \mathrm{C}$.
Results of experimental wheel tests aimed at determining the effectiveness of adding nanoclay mineral additives to oil-based cutting fluids are described in [14]. The analysis of these results as illustrated by drilling of various construction materials, has supported the idea that tribotechnological and endothermic properties of oil-based cutting fluids with nanoadditives improve. Significant increase in hardness of a drill thanks to the decrease of cutting thrust force and torque has been noted. Addition of nanoclay mineral additives to oil-based cutting fluids positively influences the formation of facings and increases the roughness of machined surfaces.

\section{Conclusion}

1. Well-known dry lubricants used as additives to oil lubricants to modify friction do not have heat-absorbing capacity, and the anti-friction and wear resistance properties of ones (graphite) are exhibited at relatively low temperatures (below $100{ }^{\circ} \mathrm{C}$ ) when sorbed water is involved in friction of their crystal lattices, while friction in the crystal lattices of others (molybdenum disulfide, serpentine) takes place in the absence of water.

2. The most important NCMA property is the capacity of its packages to be hydrowedged with a superficially occluded water at hydrogenation. As a result, an opportunity of interpackage sliding is provided due to occurring hydro-lubrication, i.e. transfer from dry to fluid interpackage friction thus improving tribotechnological and antiscoring properties of oil CLs in general.

3. From the point of view of nanoclay minerals, montmorillonite, in particular, suitability as an addition to oil CLs to increase heat removal from the cutting area, they are characterized by a higher heating capacity of water phase of the boundary layer (hydro-wedging area) comparing to volumetric water. Moreover, they possess endothermic properties, which are important for oil-based cutting fluids.

4. When CLs with NCMA is exposed to low temperatures, the broken boundary adsorption layer is automatically recovered, i.e., re-hydrated releasing some energy in the environment that is equal to the energy obtained by means of contact with a heated tool surface. The latter will be convectionally distributed in CLs volume and removed from the system into the environment.

\section{References}

1. Cutting fluids for metal cutting. Practical recommendation / Guide, (M.: NIImash, 1979)

2. D. U. Abdulgazis, Use of De-Hydration Endothermic Effect of Crystalline Hydrates to Increase a Heat-Removing Capacity of CLS on the basis of Vegetable Oils, High technologies in mechanical engineering: Collected Works NTU "KhPI", Issue 2(11), P. 3-8 (2013)

3. D.U. Abdulgazis, E.R. Ablayev, On the possibility of 
decreasing drilling temperatures by using cutting fluids with endothermic properties, Bulletin of Crimea Engineering and Pedagogical University, issue 20, pp. 30-34 (2009)

4. D.U. Abdulgazis, On methods of creating compositional vegetable oil- and crystalline hydratesbased cutting fluids, Bulletin NTU KPI. Machinery manufacturing No. 46, pp.109-110 (2005)

5. D.U. Abdulgazis, E.D. Umerov, Bench and wheel tests of effectiveness of endothermic oil-based cutting fluids, Bulletin of Crimea Engineering and Pedagogical University, issue 29, pp. 62-67 (2011)

6. W.A. Harrison, Electronic Structure and the Properties of Solids: The Physics of the Chemical Bond. - Vol.1 (translated into Russian) (M.: Mir, 1983)

7. The Mechanism of Action of Friction Modifier Molybdenum Disulphide [Digital Resource]. Access mode: http://www.avsta.ru/files/flib/35.pdf.

8. V.P. Zarubin, Development and research of tribotechnical properties of lubricants with powdered friction geomodifiers as fillers: abstract of Candidate of Sciences (PhD) thesis (Ivanovo, 2007)

9. Structural Froms and Mobility of Molecular Water in Montmorillonite [Digital Resource]. Access mode: http://chem.kstu.ru/butlerov_comm/rus/home.htm/.

10. V.V. Goryushkin, Technological Properties of Paleocene Bentonites of the Voronezh Anteclise and Opportunity of its Use, Bulleting of Voronezh University. Geology, No. 1, P.166-177 (2005)

11. V.G. Solonenko, Enhancement of Cutting Tools Working Capacity (Krasnodar, Rostov-on-Don: Kuban State Technological University, North Caucasian Department of the Academy of the Quality Problems in the Russian Federation, 1997)

12. V.G. Solonenko, M.G. Serikova, L.A. Solonenko, Working Capacity of Auger Drills (Krasnodar: Kuban State Technological University, 2004)

13. P.I. Yashcheritsin, Ye.E. Feldshtein, M.A. Kornevich, Theory of Cutting: Manual (Minsk: Novoe Znanie, 2006)

14. E.D. Umerov, D.U. Abdulgazis, U.A. Abdulgazis, Increase of hardness of a cutting tool via using environmentally friendly additives to oil-based cutting fields with endothermic and tribotechnological properties, Technical regulations in Eurasian Economic Space: collection of materials of All-Russian Conference with international participants. Yekaterinburg, pp.24-31 (2015) 